\title{
A Climatological Study of Internal Gravity Waves in the Atmospheric Boundary Layer Overlying the Brunt Ice Shelf, Antarctica
}

\author{
J. M. Rees And J. C. W. Denholm-Price* \\ School of Mathematics and Statistics, University of Sheffield, Sheffield, United Kingdom \\ J. C. King AND P. S. ANDERSON \\ British Antarctic Survey, Natural Environment Research Council, Cambridge, United Kingdom
}

(Manuscript received 7 July 1998, in final form 18 February 1999)

\begin{abstract}
Internal gravity waves are frequently observed in stably stratified regions of the atmospheric boundary layer. In order to determine the statistical influence of such waves on the dynamics of the boundary layer it is necessary to compile information concerning properties of the waves such as frequency of occurrence, propagation, and spectral characteristics. Gravity wave climatologies have been compiled from relatively few locations. In this paper a climatological study of gravity waves, in the period range 1-20 min, propagating in the stably stratified atmospheric boundary layer overlying an Antarctic ice shelf is presented. An extensive set of boundary layer measurements were compiled throughout 1991. Surface pressure fluctuations were recorded from a spatial array of six sensitive microbarographs. Wind and temperature records from an instrumented mast were also available. A beam-steering technique has been used to determine wave parameters from the surface pressure data. The microbarographs detected the presence of internal gravity waves throughout the observational campaign. Rootmean-square pressure values were typically in the region $16-40 \mu \mathrm{b}$, but a significant number of isolated events with amplitudes of up to $180 \mu \mathrm{b}$ were also found. Wave properties have been studied in conjunction with the mean wind and temperature profiles in the boundary layer. It was found that most of the wave activity did not originate locally, but from shear layers aloft, or, more commonly, from the katabatic flow regime where the ice shelf joins the Antarctic continent.
\end{abstract}

\section{Introduction}

The stably stratified (or nocturnal) boundary layer (SBL) typically develops as a result of radiative surface cooling during the night. Fluxes are weak and intermittent in the SBL and its statistics are poorly defined. Consequently, the dynamics of the SBL are less well understood than those of its convective counterpart. In midlatitudes the SBL is relatively short lived (compared to polar regions) due to diurnal effects. In polar regions the nocturnal boundary layer may persist over several months during periods of winter darkness; thus Antarctica has been chosen as the venue for several observational campaigns of the SBL in recent years (King et al. 1989; Egger et al. 1993).

\footnotetext{
* Current affiliation: School of Mathematics, Kingston University, Kingston upon Thames, United Kingdom.

Corresponding author address: Dr. Julia M. Rees, School of Mathematics and Statistics, Hicks Building, Hounsfield Road, University of Sheffield, Sheffield S3 7RH, United Kingdom. E-mail: j.rees@sheffield.ac.uk
}

Internal gravity (or buoyancy) waves (IGWs) are characteristic of the SBL. They arise due to the effect of gravity on density inhomogeneities. IGWs in the atmosphere may be generated by several mechanisms, including topographic forcing, dynamical instabilities, or wave-wave interactions. They are manifested as fluctuations in the velocity, pressure, and temperature fields. In the boundary layer, wave periods range from a few minutes to about one hour and typical wavelengths extend from about $100 \mathrm{~m}$ to several kilometers. Largeamplitude or solitary gravity waves may be formed at the head of gravity currents or fronts. Few papers have been published that present a climatology of gravity wave activity (Bull et al. 1981; Gedzelman 1983; Einaudi et al. 1989). Further studies of the spatial and temporal characterisitics of IGWs are required for the evaluation of their statistical influence on boundary layer dynamics. Detailed knowledge of the properties of such waves is needed for the development of parameterization schemes for use in weather forecasting and pollution dispersion models.

Internal gravity waves are best observed via surface pressure fluctuations (Anderson et al. 1992). Accordingly, a number of observational campaigns have in- 
cluded the deployment of an array of microbarographs. One of the first such campaigns is reported in Hooke et al. (1972). An array of four microbarographs was used to provide wave amplitude, horizontal phase speed, direction, and wavelength in an attempt to characterize wavelike structures observed on acoustic sounder records. Keliher (1975) compared the occurrence of gravity waves as detected by an array of microbarographs with the existence of dynamically unstable wind shear layers at Boulder, Colorado, and Washington D.C. He found that over half of the gravity wave events detected were shear induced. Merrill (1977) used data from a meteorological mast and microbarograph array to study shear instability in the airflow near the ground under stable conditions. Cheung and Little (1990) used a microbarograph array in conjunction with an instrumented mast and sodar to study waves at the Boulder Atmospheric Observatory. Einaudi et al. (1989) operated an array of eight microbarographs for one month at the same site as part of a climatological study of gravity waves and other coherent disturbances detected in the boundary layer. They observed highly coherent structures in the period range 1-20 min most of the time. For disturbances with periods of up to $5 \mathrm{~min}$, a relationship was found between the turbulent kinetic energy and the root-mean-square (rms) pressure field at the ground. Such a relationship was not found for the longer scales indicating that these modes were influenced by a deeper atmospheric zone. More recently, Egger et al. (1993) described two gravity wave events detected at the George von Neumayer Station in Antarctica using a 42-m mast and an array of four microbarographs spaced approximately $1.7 \mathrm{~km}$ apart. The events were selected for analysis as the wave frequency was well defined in each case. One event was classified as belonging to the class of trapped, neutral modes; the other was shown to be a wave propagating on a surface inversion.

A mesoscale network comprising four microbarographs has been in operation in southern Germany since 1992 (Hauf et al. 1996). They applied a wavelet filter to the data obtained from each sensor in order to isolate gravity wave events from background fluctuations. They identified periods dominated by sinusoidal fluctuations caused by gravity wave activity. Such events occurred in packets of at most five wavelengths and were associated with convective processes. Most of the background signals were characterized by well-correlated irregular shaped pressure fluctuations with amplitudes of several microbars. These were associated with the existence of drifting density inhomogeneities.

In Antarctic regions the propagation of internal gravity waves is possible throughout the year as the boundary layer is nearly always stably stratified. Internal gravity waves have been studied at a number of sites, both on the Antarctic plateau (Kikuchi 1988) and in coastal regions (King et al. 1987; Egger et al. 1993). Occurrences of both trapped, neutral modes (Rees and Mobbs

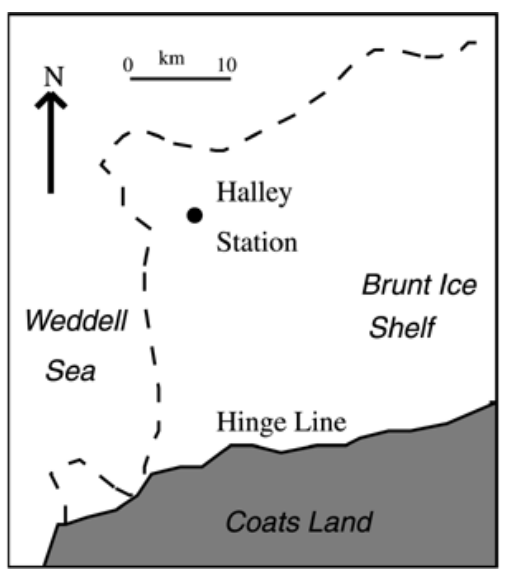

FIG. 1. Sketch map showing the Halley IV station site.

1988) probably caused by topographic effects and waves originating from shear instabilities (King et al. 1987) have been reported. In contrast to the study of Hauf et al. (1996), ground-based instruments always detect the presence of IGWs at Halley station, Antarctica, when the surface layer is stably stratified. This paper focuses on observations of the SBL made at Halley IV station, Antarctica. The motivation for this project arose from the need to build a comprehensive dataset of boundary layer observations compiled over an extended period of time. This new dataset has provided the basis for developing a climatology of gravity wave activity at Halley. This climatology will be of relevance for future studies on boundary layer fluxes, transport processes, and mixing.

\section{a. The STABLE project}

The Brunt Ice Shelf, Antarctica, is an ideal location for studying the SBL (Fig. 1). The 3-month period of darkness during the austral winter allows observations to be made that are relatively free of complicating orographic and diurnal effects. Halley is situated toward the seaward edge of the ice shelf. The ice shelf slopes downward toward the coast with a mean gradient of 1:2000. A uniform fetch of about $40 \mathrm{~km}$ extends from Halley to the region of irregular terrain known as the Hinge Line, where the ice shelf joins the interior plateau. In the vicinity of the Hinge Line the Antarctic interior is $1600 \mathrm{~m}$ above the ice shelf. In 1986 and 1991 the British Antarctic Survey ran two intensive observational campaigns to monitor the SBL overlying the Brunt Ice Shelf from their base at Halley IV station $\left(76.6^{\circ} \mathrm{S}\right.$, $26.7^{\circ} \mathrm{W}$ ). The project was called the Stable Antarctic Boundary Layer Experiment and given the acronym STABLE. We will refer to the two phases of the campaign as STABLE I and STABLE II, respectively.

The instrumentation available during STABLE I included a 32-m meteorological mast with Kaijo-Denki ultrasonic thermo-anemometers deployed at nominal 
heights of 5, 16, and $32 \mathrm{~m}$. Data from the sonics were used to study turbulence (King 1990) and IGWs in the SBL. These were supplemented by a number of wind vanes, cup anemometers, and platinum resistance thermometers to provide vertical profiles of the wind and temperature fields. A monostatic sodar coupled with daily radiosonde ascents provided information on the structure of the lower troposphere up to $3 \mathrm{~km}$ (King 1989). Two remote masts, each with a wind vane and cup anemometer mounted at $8 \mathrm{~m}$, were positioned so as to form a 200-m triangle with the main mast. A limited number of wind observation records from this triangular array allowed some initial estimates of the spatial and temporal properties of the waves to be made using a correlation technique (Rees and Mobbs 1988). Results from this study indicated that most of the fluctuations in the lowest $8 \mathrm{~m}$ corresponded to horizontally propagating neutral waves with a trapped modal structure in the vertical. The majority of the waves were found to propagate from a southeasterly direction at phase speeds in the range $2-6 \mathrm{~m} \mathrm{~s}^{-1}$. The direction of wave propagation was veered by $40^{\circ}$ from the mean wind direction at $8 \mathrm{~m}$. An explanation for this phenomenon was suggested based on the assumption that the waves were formed in the vicinity of the Hinge Line, which is an area of steeply sloping terrain where katabatic effects are marked. Waves generated in the region of katabatic flow would propagate along the direction of the mean slope, that is, from the southeast. The Coriolis force would cause deflection of the wind direction across the ice shelf (Rees and Mobbs 1988). However, although calculations indicate that over a fetch of $40 \mathrm{~km}$, one would expect to observe a deflection of about $40^{\circ}$, recent observations from an automatic weather station deployed $10 \mathrm{~km}$ from the Hinge Line showed that the wind direction in this region was the same as that at Halley (King 1993). This apparent contradiction implies that other factors-for example, mesoscale effects such as frontal systems-influence the wind regimes across the ice shelf. Although providing a useful insight into the wave activity at Halley, the dataset available for the study by Rees and Mobbs (1998) was restricted due to the extreme climatological conditions which prevail at Halley. At times the anemometers and wind vanes were severely damped due to riming. This provided the motivation for a second intensive campaign to investigate the properties of IGWs in the boundary layer at Halley using more robust instrumentation.

It was demonstrated by Anderson et al. (1992) that for a stably stratified boundary layer with a mean velocity profile exhibiting a near-surface jet (such a scenario is typically observed at Halley), the pressure signature of a trapped wave close to the surface attains a large value at the surface, whereas the amplitudes of vertical velocity and buoyancy acceleration are small at the surface. Thus IGWs are best detected via measurements of surface pressure fluctuations. Surface pressure sensors respond to fluctuations in the total mass of air

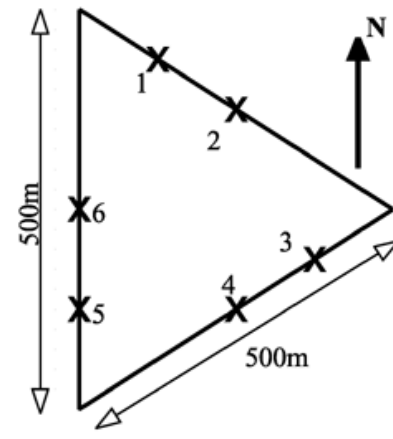

Microbarograph array

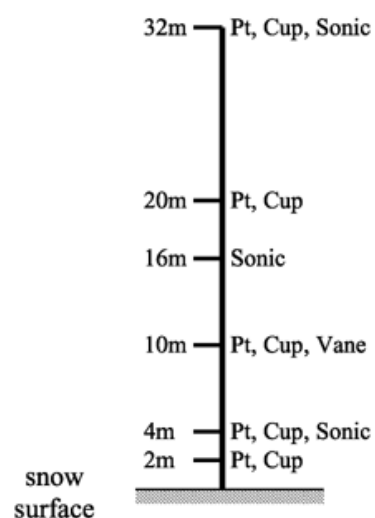

Instrumented mast
FIG. 2. Instrumentation used in the STABLE II experiment.

in a column above it. A sensitive microbarograph was developed specifically to withstand the climatological conditions at Halley (Anderson et al. 1992). The microbarograph was designed to operate in conditions of permanent snow cover at temperatures as low as $-50^{\circ} \mathrm{C}$. A micromanometer was used to measure the pressure differences between the atmosphere and a fixed reference volume that was held at a constant pressure. The micromanometer was vented to the atmosphere via a network of static heads buried $1 \mathrm{~m}$ under the snow. (The snow cover rose to a depth of $1.54 \mathrm{~m}$ by the end of the campaign; the accumulation of snow was accounted for in the subsequent data analysis routines.) This arrangement had the advantage of filtering out spurious signals induced by fluctuations in wind speed. Using a simple theory based on D'Arcy's model for flow through a porous medium, Anderson et al. (1992) showed the layer of snow covering the static heads does not adversely affect the pressure fluctuations associated with the IGWs of interest. The amplitude of a wavelike pressure signal propagating with a phase speed greater than $2 \mathrm{~m} \mathrm{~s}^{-1}$ at a frequency less than $0.02 \mathrm{~s}^{-1}$ is virtually unaffected by this layer of snow (the attenuation is less than 5\%).

An array of six microbarographs was built for the project, which formed part of the STABLE II campaign and ran from February through November 1991. The layout of the microbarograph array is shown in Fig. 2, together with a schematic of the instrumented mast that was operated in conjunction with the array during STABLE II. The layout of the array was chosen so as to optmize the data analysis procedures used. This is discussed further in the following section. Pressure fluctuations were measured over a period of 9 months in 1991. The microbarographs were sampled every second. Data were logged in the form of 10 -s averages. Data from the mast instruments were recorded in a similar manner. Root-mean-square pressure amplitudes (calculated over periods of approximately $3 \mathrm{~h}$ ) varied between 10 and $140 \mu \mathrm{b}$ after removal of the mean trend generated by the passage of synoptic weather systems 
[see Denholm-Price and Rees (1998) for a description of this procedure]. It is not possible to distinguish a priori between pressure signals generated by traveling waves and propagating density fluctuations that remain coherent across the array (width $\sim 500 \mathrm{~m}$ ). However, analysis of the cross-spectral phase (discussed below in section 2) suggests that the great majority of signals detected by the array are related to internal gravity waves. Consequently, the term "waves" will be used to refer to all wavelike signals obtained by the beamsteering analysis of data from the array.

\section{b. Analysis of data from a spatial array of sensors}

The extent of the array was chosen so as to be appropriate for the detection of typical wavelengths and phase speeds of the Halley gravity waves as predicted by Rees and Mobbs (1988). The layout of the sensors was chosen in order to obtain optimal results for wave phase speeds and directions using a beam-steering procedure. Haubrich (1968) showed that an optimal layout for an array of six sensors is as shown in Fig. 2. The array is isometric and the array transfer function approximates a delta function at the origin of wavenumber-wavenumber space. Thus on forming the convolution of the array transfer function with data from the sensors, the displaced position of this central maximum is usually well defined. The displacement of the central maximum can be related to the speed and direction of a wave propagating in the plane of the array. A number of authors (including Rees and Mobbs 1988; Einaudi et al. 1989) have used a cross-correlation technique in order to extract wave parameters from a spatial array of sensors. The beam-steering method used herein has a number of advantages over schemes based on routine correlation techniques.

1) The algorithm employed here is able to identify multiple wavelike signals with different wavenumbers at the same frequency. This is of particular benefit when considering atmospheric gravity waves. It is possible for modes generated by topography and shear in different regions of the atmosphere to be observed simultaneously.

2) The method produces more accurate results over a range of frequency and wave parameters.

The beam-steering procedure used here is outlined in the following section, but a detailed account of the method can be found in Denholm-Price and Rees (1999, henceforth DPR99).

The procedures used to analyze data from the mast instruments as well as those from the microbarograph array are described in section 2. A case study using observations from 3 May 1991 is presented as an illustration. The mean atmospheric conditions prevalent at Halley are summarized in section 3, and a climatology of the wave activity in the overlying boundary layer is presented in section 4 . An attempt to classify the waves is proposed in section 5. The results are summarized in section 6.

\section{Data analysis routines}

The two main methods used for processing data from the STABLE II dataset are based on spectral routines. A beam-steering algorithm was used to determine wave speed, propagation direction, and wavelength from the pressure records from the microbarograph array. Crossspectral analysis was used to determine the nature of the wavelike fluctuations. Data were logged in the form of 10-s averages throughout the campaign. Records were split into blocks of 1024 points ( $\sim 2 \mathrm{~h}$ and $50 \mathrm{~min}$ ) for analysis. A number of parameters are obtained using the beam-steering and other spectral methods for propagating disturbances detected within each block.

\section{a. The beam-steering procedure}

The term "beam-steering" is used to describe any process that uses data from a spatial array of sensors to determine the direction from which a disturbance is propagating. Conceptually, the "beam" of the array is rotated through the azimuth of interest until a maximal signal is found. The phase difference (or time lag) between the signal at different locations within the array provides the information necessary to estimate wave properties such as phase speed and propagation direction.

The beam-steering algorithm used in this study is described fully in DPR99 and references contained therein. It produces estimates for parameters describing the wavelike disturbances measured by the microbarograph array. The pressure data are dominated by synoptic variations of the order of 20-1000 Pa occurring over timescales of a few hours. Typically there exists a spectral gap between the synoptic signal responsible for the mean trend in the data and the IGWs of interest. Data from the array are processed by first subtracting the mean and then a polynomial trend (Denholm-Price and Rees 1998). The data were then detrended further using a low-pass filter with a cutoff frequency of $f_{\text {cut }}$ $=0.00049 \mathrm{~s}^{-1}$ (a period of $\sim 34 \mathrm{~min}$ ). This cutoff frequency was chosen so as to take advantage of the spectral gap.

Estimates of wave parameters derived from the spectral data correspond with certain maxima of the crosspower spectral density function, $P(f, \mathbf{k})$, where $f$ is the frequency and $\mathbf{k}$ is the wavenumber. The so-called "high-resolution" discrete estimate of this continuous function is used, denoted by $P^{\prime}\left(f_{n}, \mathbf{k}\right)$ (Capon 1969). Estimates for wave parameters are determined from the position in the wavenumber plane of maxima in $P^{\prime}$ at a given frequency $f_{n}\left(n=1, \ldots, N_{b}\right)$, which is the center of a finite-width frequency bin. The estimated maxima are denoted $\mathbf{k}_{j}, j=1, \ldots, J$, for there may be more than one signal detected within each frequency 
bin. The beam-steering algorithm determines the "best" out of the estimates in each frequency bin using a combination of the wave-adjusted squared cross coherence, $\mathrm{coh}^{2}$; Fisher statistic, $F$; and various limits that are determined by the array geometry (discussed further in DPR99). The direction of propagation of the detected disturbance, $\theta$; its phase speed, $c$; and wavelength, $\lambda$, are then given by Eq. (1):

$$
\lambda=\frac{2 \pi}{\left|\mathbf{k}_{0}\right|}, \quad c=\lambda f_{0}, \quad \text { and } \quad \theta=\arg \left(\mathbf{k}_{0}\right) .
$$

The beam-steering obtains estimates of $c, \theta$, and $\lambda$ for $N_{b}=33$ frequency bins, ranging from $f_{1} \approx 0.0008$ to $f_{33} \approx 0.023 \mathrm{~s}^{-1}$ (linearly spaced). These correspond to periods of approximately $20 \mathrm{~min}$ to $60 \mathrm{~s}$, respectively, which encompass the range of periods expected for internal gravity waves (Einaudi et al. 1988; Rees and Mobbs 1988).

The geometry of the array as pictured in Fig. 2 was chosen to maximize the ability of the array to resolve signals in $P^{\prime}$ while minimizing sidelobe leakage (Haubrich 1968). The minimum spacing of the instruments determines the largest wavenumber that can be detected, which is $k \approx 0.03 \mathrm{~m}^{-1}$. A larger value of $k_{\max }=0.04$ $\mathrm{m}^{-1}$ can be accommodated with additional processing (see DPR99 and below, for discussion of aliased estimates). The array diameter and sampling rate set an upper limit of $25 \mathrm{~m} \mathrm{~s}^{-1}$ on the phase speeds that can be detected reliably by the beam-steering.

The Fisher statistic, $F$, is used to distinguish between "good" and "bad" signals. Following the theory of Smart and Flinn (1971), it can be shown that for an array of six sensors a wave estimate corresponds to the central maximum of the cross-power spectral density function, rather than to a sidelobe, if the Fisher statistic is greater than 4 . Consequently a signal with $F<4$ is assumed to be unreliable and hence is removed from further consideration. This corresponds to a minimum signal-to-noise ratio, $\mathrm{SN}$, of $-12 \mathrm{~dB}$ as $F$ is related to SN by

$$
F\left(f_{n}\right)=\left(N_{\mu}-1\right) \times \mathrm{SN}\left(f_{n}\right)
$$

where

$$
\mathrm{SN}\left(f_{n}\right)=\frac{P_{\mathrm{det}}\left(f_{n}\right)}{P_{\mathrm{tot}}\left(f_{n}\right)-P_{\mathrm{det}}\left(f_{n}\right)},
$$

where $N_{\mu}=6$ is the number of instruments in the array, $P_{\text {tot }}\left(f_{n}\right)$ is the total spectral power in bin $n$, and $P_{\text {det }}\left(f_{n}\right)$ $=\hat{P}\left(f_{n}, \mathbf{k}_{j}\right)$ is the detected spectral power at wavenumber $\mathbf{k}_{j}$ (i.e., for estimate $j$ ). The $\hat{P}$ is the low-resolution discrete estimate of $P$ (Capon 1969) and is used here because it is more closely related to the spectral power than $P^{\prime}$.

\section{b. Cross-spectral analysis}

The cross-spectrum between pairs of instruments, including those from the mast and microbarograph 1,
TABLE 1. Table showing cross-spectral phase relationships. Here, " $\operatorname{sgn}(c-\bar{u})$ " indicates a "+" when $c>\bar{u}$ and "-" when $c<\bar{u}$ ( $c$ is the phase speed and $\bar{u}$ the mean wind speed); "- " implies that the phase is indeterminate.

\begin{tabular}{llcc}
\hline \hline Phase & \multicolumn{1}{c}{$\begin{array}{c}\text { Internal gravity } \\
\text { waves }\end{array}$} & $\begin{array}{c}\text { Kelvin-Helmholtz } \\
\text { instability }\end{array}$ & Turbulence \\
\hline $\mathrm{Ph}(u p)$ & $0, \pi$ & $\pm \pi$ & - \\
$\mathrm{Ph}(w p)$ & $-\operatorname{sgn}(c-\bar{u}) \pi / 2$ & $0, \pi$ & - \\
$\mathrm{Ph}(T p)$ & $0, \pi$ & $0, \pi$ & - \\
$\mathrm{Ph}(u w)$ & $\pm \pi / 2$ & - & $0, \pi$ \\
$\mathrm{Ph}(u T)$ & $0, \pi$ & - & - \\
$\mathrm{Ph}(w T)$ & $\operatorname{sgn}(c-\bar{u}) \pi / 2$ & $\pm \pi$ & $0, \pi$ \\
\hline
\end{tabular}

which is buried near to the base of the mast in Fig. 2, can be used to calculate the cross-spectral phase and coherence over the frequencies of interest. Following Rees (1991), it can be demonstrated that horizontally propagating IGWs, Kelvin-Helmholtz $(\mathrm{K}-\mathrm{H})$ instabilities, and turbulence have different cross-spectral phase "signatures." We denote the cross-spectral phase between instruments $a$ and $b$ as $\operatorname{Ph}(a b)$. The cross-spectral coherence is also evaluated and, according to Rees (1991), a value of at least 0.06 is necessary for $95 \%$ confidence in the associated phase.

The pressure signal measured by the microbarographs is a weighted average of fluctuations throughout the column of air above the instrument. It may even be possible to detect directly $\mathrm{K}-\mathrm{H}$ billows generated near the top of the SBL. For monochromatic occurrences of IGWs or $\mathrm{K}-\mathrm{H}$ instabilities, linear theory (i.e., neglecting the effects of nonlinear wave interactions and turbulence) predicts the phase relationships shown in Table 1.

Nonlinear effects are neglected throughout this study. However, to a first order, neglecting the interaction between turbulence and the detected waves, the diffusive effects of turbulence may affect such idealized signals and so the cross-spectral phases may not take such precise harmonic values, although the general relationships should be preserved. In a stably stratified boundary layer, cogradient turbulent fluxes result in a downward transport of heat $[\mathrm{Ph}(w T)=\pi]$. Countergradient fluxes $[\mathrm{Ph}(w T)=0]$ are less commonly observed (Rees 1991). Note that the assertion that $\mathrm{Ph}(w T)= \pm \pi / 2$ and $\mathrm{Ph}(u w)$ $= \pm \pi / 2$ implies that IGWs are not responsible for the vertical transport of heat and momentum. This is true in the linear limit only. When nonlinear effects are important-for example, when wave-wave or wave-turbulence interactions are important or when wave breaking occurs - this will not hold. A study of the influence of gravity waves on the boundary layer fluxes at Halley is being undertaken.

\section{c. Example of results from a specific section of data}

An example of output from all six microbarographs is shown in Fig. 3, taken from around 1200 UTC on 3 May 1991. The data have been detrended and then dis- 


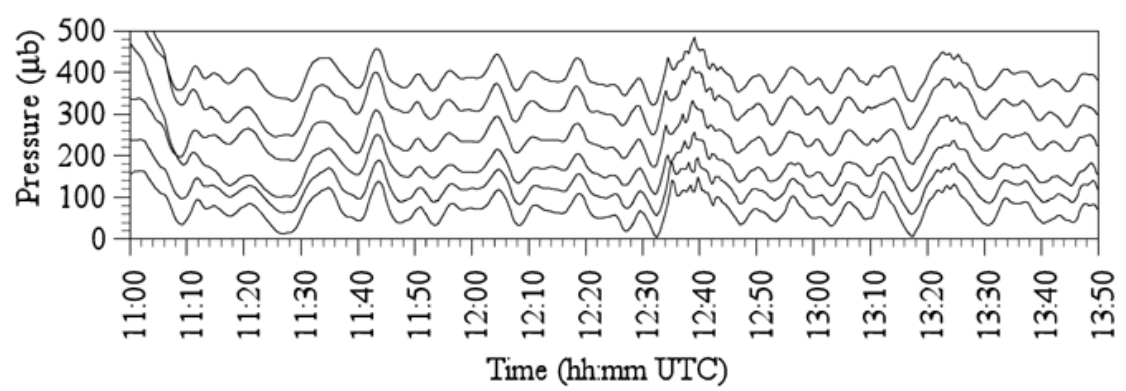

FIG. 3. Data from six microbarographs from 3 May 1991.

placed by a constant value to separate the individual records in the figure. We can clearly see periodic fluctuations with peak-to-peak amplitude of $\sim 30-60 \mu \mathrm{b}$ throughout the record. It is evident that the disturbances remain coherent across the array. Small phase shifts between instruments (e.g., as indicated by the position of the peak at $\sim 1145$ UTC) show that the disturbance is propagating across the array, and the varying period of the signal indicates the presence of a number of harmonics. There is also a burst of fluctuations with a shorter period after 1230 UTC that lasts for just $10 \mathrm{~min}$.

\section{1) BEAM-STEERING RESULTS}

Results from the beam-steering for the 1024-point block of data from 1100:06 to 1350:36 UTC are shown in Figs. $4 \mathrm{a}, \mathrm{b}$ and $5 \mathrm{a}-\mathrm{c}$. In all figures the best estimates found by the beam-steering are indicated by asterisks (*). The solid lines show results associated with the main peak in each frequency bin. The algorithm chooses best estimates that correspond with peaks in the spectral power. This is illustrated in Fig. 4a by the solid line which shows the spectral amplitude. It is evident that

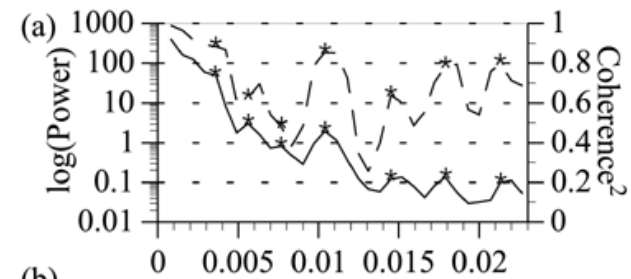

(b)

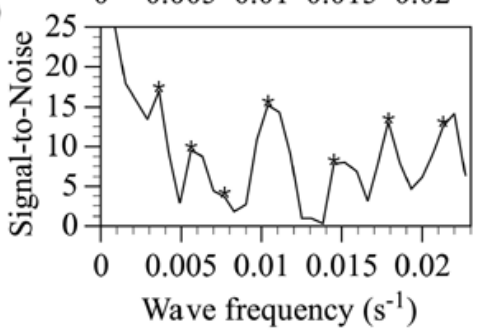

FIG. 4. Beam-steering results from 3 May 1991: Power, coherence, and signal-to-noise. Frequencies for which wave parameters were determined using the beam-steering algorithm are indicated by *. In (a), the solid line denotes spectral amplitude and the dashed line denotes squared coherence. the asterisks correspond with peaks in the amplitude (solid line). They are also associated with peaks in the squared coherence, illustrated by the dashed line. In Fig. $4 \mathrm{~b}$ the signal-to-noise ratio $\mathrm{SN}$ is plotted. We can see that $\mathrm{SN}$ varies from $\sim 17$ to $4 \mathrm{~dB}$. According to DPR99, for a wave near the central frequency of the lowest bin, this range of $\mathrm{SN}$ corresponds to errors in wind direction of $\pm 20^{\circ}$ and in phase speed of $\pm 20 \%$. If the wave frequency is offset from the center by an amount $\Delta f$, then the phase speed measured by the array will differ from the true value by an amount dependent on the frequency offset, that is, $\Delta c / c=\Delta f / f$, since the beamsteering measures the wavenumber from which $c$ is calculated. With the current linear bins the maximum frequency error is $\Delta f=0.00034 \mathrm{~s}^{-1}$. The maximum percentage error in phase speed therefore varies from

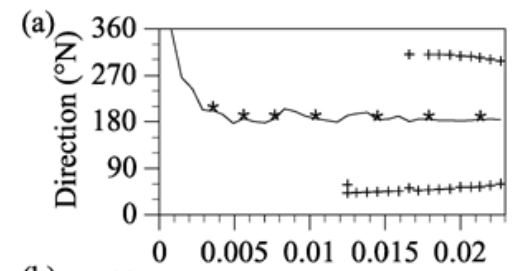

(b)

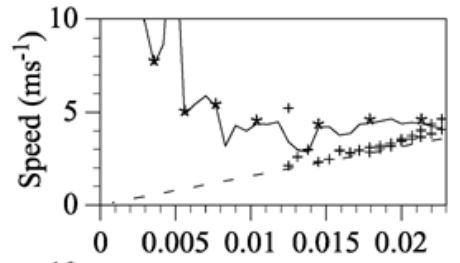

(c)

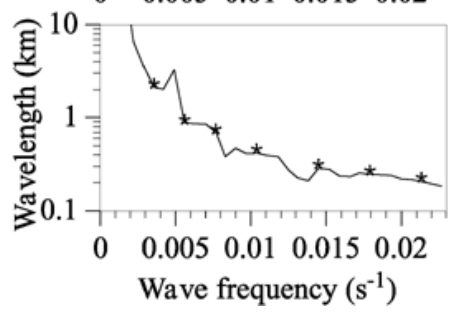

FIG. 5. Beam-steering results from 3 May 1991: (a) direction, (b) phase speed, and (c) wavelength. Frequencies for which wave parameters were determined using the beam-steering algorithm are indicated by $*$. In (a) and (b) the plus symbols $(+)$ represent aliased estimates. In (b) the dashed line indicates the lowest limit of estimates for phase speed. 
(a)

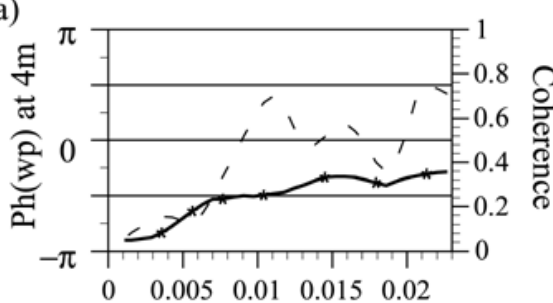

(c)

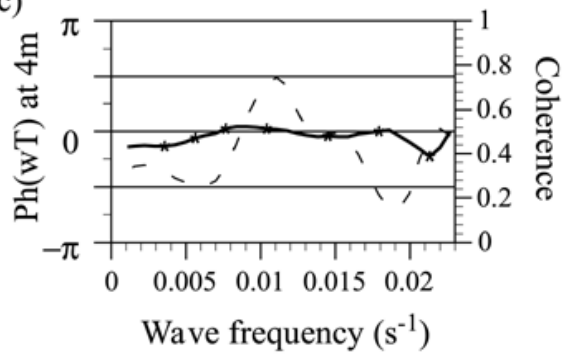

(b)

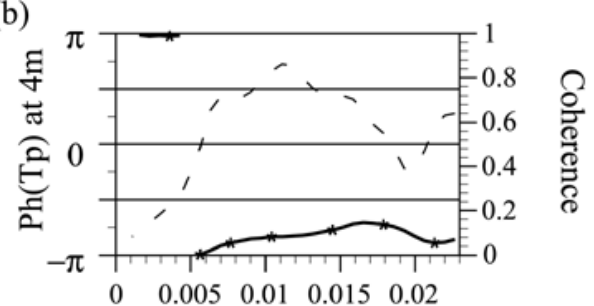

(d)

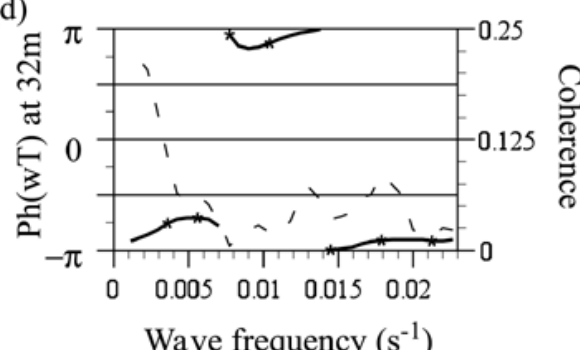

FIG. 6. Beam-steering results from 3 May 1991: Cross-spectral phase and coherence. Frequencies for which wave parameters were determined using the beam-steering algorithm are indicated by *. Cross-spectral phases are indicated by solid lines and the coherences by dashed lines.

$\sim 43 \%$ to $\sim 1.5 \%$ from the lowest $\left(f=0.0008 \mathrm{~s}^{-1}\right)$ to highest $\left(f=0.023 \mathrm{~s}^{-1}\right)$ frequency bins.

In Fig. 5a the beam-steering propagation direction is plotted against frequency. The beam-steering predicts that the waves at all frequencies $f \geq 0.003 \mathrm{~s}^{-1}$ propagate from $\theta \sim 180^{\circ}$. In this case, estimates with $f<0.003$ $\mathrm{s}^{-1}$ are rejected as they correspond to phase speeds in excess of $25 \mathrm{~m} \mathrm{~s}^{-1}$ or because they are inconsistent with adjacent frequency bins (this procedure was discussed further in DPR99). The minimum frequency corresponds with a period of $\sim 5 \mathrm{~min}$.

The plus $(+)$ symbols in Fig. 5a represent estimates that were rejected by the beam-steering program. In this case it is evident that the rejected estimates are geometrically aliased to the best estimates (discussed in DPR99). The maximum wavenumber $k_{\max }=0.04 \mathrm{~m}^{-1}$ corresponds to a minimum phase speed $c_{\min }=2 \pi f / k_{\max }$. This is indicated by the dashed straight line in Fig. $5 b$ that forms the lower boundary of the estimates for phase speed. Some additional analysis is required to characterize waves propagating at phase speeds close to $c_{\min }$ aliased estimates are observed in the cross-power spectral density function that are related to the "correct" estimate. It is not possible to immediately identify the correct estimate as they have identical values of $F$ and similar $\operatorname{coh}^{2}$. If aliased estimates occur the correct estimate is taken to be the one whose propagation direction most closely matches that of a good estimate detected without aliased partners in an adjacent frequency bin. If there are no matching estimates, then all of the aliased estimates within a frequency bin are rejected. This procedure allows slower waves to be detected than would otherwise be possible.

The ability of the current method to detect a wide range of waves is further illustrated by considering the detected wavelength, shown in Fig. 5c. The array diameter is $\sim 500 \mathrm{~m}$. By taking "snapshots" of the wave field, the maximum detectable wavelength would be half this. However, because the beam-steering method uses blocks of data it essentially observes the passage of wave fronts across the array. For example, the maximum wavelength detected in the current example is $2125 \mathrm{~m}$, over four times the diameter of the array.

\section{2) CRoss-Spectral PHASE Results}

In Fig. 6 the cross-spectral phase and squared coherence, $\operatorname{coh}^{2}$ (dashed line, right-hand axis), are plotted against frequency. Again, the best beam-steering estimates are marked with asterisks. In Fig. 6a, $\mathrm{Ph}(w p)$ (using $w$ from the 4-m sonic anemometer on the mast in Fig. 2) is plotted. We can see that where $\operatorname{coh}^{2}$ is greater than 0.1 the phase is $\sim-\pi / 2$. This is consistent with the detected waves being IGWs traveling faster than the mean wind speed (Table 1). In this case the phase speed is $\sim 5 \mathrm{~m} \mathrm{~s}^{-1}$ and the wind speed at $4 \mathrm{~m}$ is $\approx 3.8 \mathrm{~m} \mathrm{~s}^{-1}$. Phase $\mathrm{Ph}(T p)$ ( $T$ also from the $4-\mathrm{m}$ sonic anemometer) is plotted in Fig. 6b. While $\operatorname{coh}^{2}$ is large for $f>0.005 \mathrm{~s}^{-1}$, the phase is $\sim-3 \pi / 4$. This deviation from the values of $\pm \pi$ associated with linear IGWs may arise if the amplitudes of the observed waves exceed those for which linear theory is strictly applicable.

In Figs. 6c, $\mathrm{d} \mathrm{Ph}(w T)$ is plotted using the sonic anemometers at 4 and $32 \mathrm{~m}$, respectively. At $4 \mathrm{~m} \mathrm{coh}^{2}$ is greater than 0.1 at all frequencies and the phase is consistently close to zero, indicating that there is a net transport of heat. At $32 \mathrm{~m} \mathrm{coh}^{2}$ is quite small (note the different axis scales), suggesting that the results may be unsatisfactory. However, there is a suggestion that the $\mathrm{Ph}(w T)$ at $32 \mathrm{~m}$ is $\pm \pi$ rather than 0 as found nearer to 


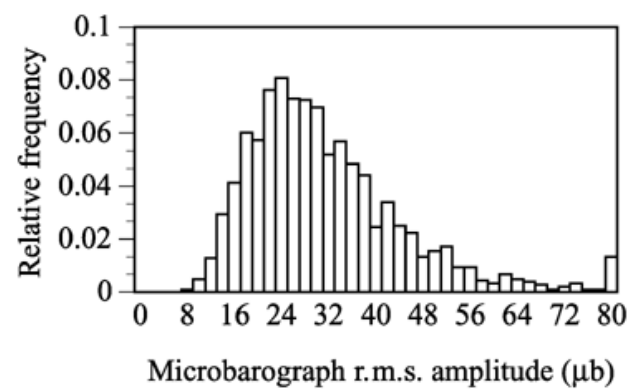

FIG. 7. Mean rms pressure from 1024-point blocks of detrended data.

the surface. At $4 \mathrm{~m}$ fluctuations in $w$ and $T$ are in phase and there is a strong, stable temperature gradient that is consistent with a negative heat flux (toward the snow surface). At $32 \mathrm{~m}$ the temperature gradient is much smaller and may even be unstable (being close to zero, its sign is easily changed by the data used and by errors in the instruments). In this case any heat flux caused by turbulent motions will be small, hence the low coherence in Fig. 6d. The gradient Richardson number $\mathrm{Ri}_{g}$ is defined as

$$
\mathrm{Ri}_{g}=\frac{g}{\theta_{0}} \frac{\left(\frac{d T}{d z}+\frac{g}{c_{p}}\right)}{\left[\left(\bar{u} \frac{d a}{d z}\right)^{2}+\left(\frac{d s}{d z}\right)^{2}\right]},
$$

where $g \approx 9.81 \mathrm{~m} \mathrm{~s}^{-2}$ is the acceleration due to gravity; $\theta_{0}$ a reference temperature; $c_{p} \approx 1010.0 \mathrm{~m} \mathrm{~s}^{-2}$ is the specific heat at constant pressure; $T$ is the temperature $\left({ }^{\circ} \mathrm{C}\right) ; s$ and $a$ are the mean wind speed and direction, respectively; and $z$ is height above the snow surface. The vertical profile of $\mathrm{Ri}_{g}$ (not shown) has a large value $\left(\mathrm{Ri}_{g} \sim 6\right)$ at $12 \mathrm{~m}$, with smaller, positive values at 4 and $32 \mathrm{~m}$. Thus it is unsurprising that we measure a turbulent signature at these levels, rather than a wavelike phase of $\pm \pi / 2$ for $\mathrm{Ph}(w T)$.

In the Antarctic the SBL is very shallow, often less than the height of the mast due to the strong stable stratification (King 1989). Consequently, the usual loglinear profiles of wind speed and temperature are often inappropriate when fitting curves to the mast data up to $32 \mathrm{~m}$. Therefore, in subsequent analyses, profiles of $\mathrm{Ri}_{g}$ are replaced by bulk measurements, derived directly from the differences between the mean values of wind speed, direction, and temperature at 4, 10, and $32 \mathrm{~m}$ (using data from the 4- and 32-m sonic anemometers and the 10-m vane, anemometer, and platinum thermometers). This leads to values for a bulk Richardson number, denoted $\mathrm{Ri}_{b}$, evaluated between $32-10 \mathrm{~m}$ and $10-4 \mathrm{~m}$. In this case the "32-m" value of $\mathrm{Ri}_{b}=1.1$ and the " $10-\mathrm{m}$ " value is 2.5 . These are comparable with average values over the same heights derived by fitting curves to vertical profiles of wind speed, direction, and

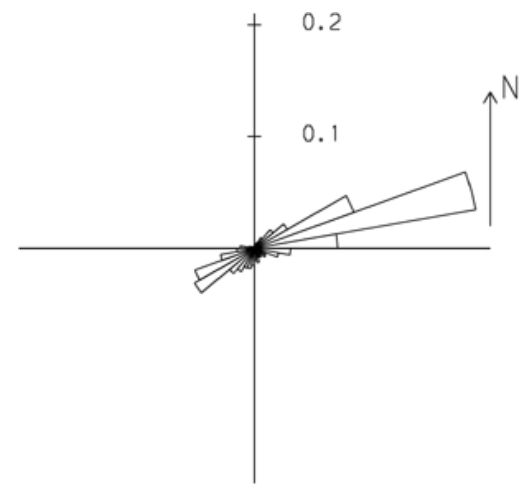

FIG. 8. Mean wind direction at $32 \mathrm{~m}$.

temperature, which avoids the problem of deciding which functions to fit the data to. We will therefore usually measure the stability of the SBL in terms of the Brunt-Väisälä frequency.

\section{Summary of mean atmospheric conditions at Halley IV station}

King (1989) describes the mean conditions at Halley in 1985. In the 1991 STABLE II dataset similar conditions are observed. Mean atmospheric conditions are calculated from 1024-point blocks, corresponding with those processed in the beam-steering analysis. Throughout the year, the microbarographs detected wavelike fluctuations. After removing the mean trend generated by synoptic weather systems (discussed in DenholmPrice and Rees 1998) a histogram of the rms pressure measured by microbarograph 1 is presented in Fig. 7. The most common fluctuations measured had rms amplitudes of $24 \mu \mathrm{b}(10 \mu \mathrm{b}=1 \mathrm{~Pa})$, but isolated events with amplitudes up to $180 \mu \mathrm{b}$ were detected. There is no noticeable variation in pressure amplitude with time (such as between the austral winter and summer). The largest-amplitude events seem to occur during the winter months, but this may be misleading as relatively fewer observations were made in the summer (the experiment ran from May to November 1991).

Figure 8 contains a polar histogram showing the mean wind direction at $32 \mathrm{~m}$ (from the $32-\mathrm{m}$ sonic anemometer in Fig. 2) in the usual meteorological sense. The predominant wind direction is from $\sim 80^{\circ}$, which is the direction of the Antarctic interior from Halley (henceforth the wind is referred to as "offshore" for directions $45^{\circ}-180^{\circ}$ ). A secondary peak corresponds with wind from the sea ("onshore," clockwise from $190^{\circ}$ to $45^{\circ}$ ). From the 9 months of observations taken during STABLE II there were 937 blocks of data with offshore mean winds at $32 \mathrm{~m}$, and 549 onshore blocks from a total of 1486 blocks with valid data from the $32-\mathrm{m}$ sonic.

Histograms of the mean wind speed at $32 \mathrm{~m}$ are shown in Fig. 9. In this figure relative frequencies are plotted for the two categories (normalized so that the sum of 


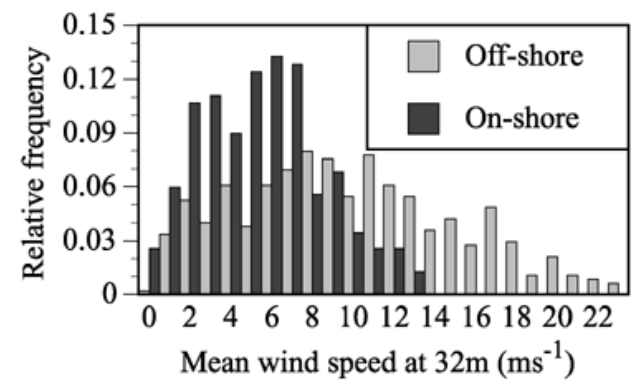

FIG. 9. Mean winds at $32 \mathrm{~m}$ for offshore and onshore categories.

frequencies in each category is 1). Onshore winds tend to be significantly slower than offshore. This is because easterly winds result from synoptic and topographic (katabatic) forcing acting together. Westerly winds occur when these forcings are opposed (King 1989). Relatively slower onshore winds tend to coincide with conditions of stronger stability. This is illustrated in Fig. 10 where histograms of the bulk Brunt-Väisälä frequency are plotted for the two cases.

\section{Wave climatology}

As mentioned in the introduction, the primary purpose of compiling a gravity wave climatology is to provide a foundation for studies aimed at assessing the influence of gravity waves on fluxes in the boundary layer. Yägue and Cano (1994) showed that local scaling hypotheses fail to adequately describe turbulent transfer of heat and momentum at Halley due to the presence of gravity waves. Hence in this study, in addition to compiling a gravity wave climatology, we have sought to categorize the wave activity at Halley in relation to the prevailing winds and thermal stability. The results obtained will aid further studies on turbulent transfer processes in the SBL at Halley.

By applying the beam-steering program to all nonoverlapping, 1024-point data blocks available from the STABLE II project, a dataset containing estimates of wave parameters was compiled. From a total of 1828 such blocks, 13688 wave parameters were obtained. An average of 7 distinct wave modes were identified from

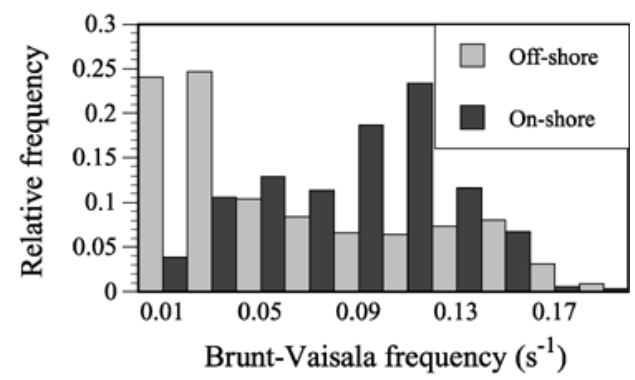

FIG. 10. The 10-m bulk Brunt-Väisälä frequency for offshore and onshore winds.

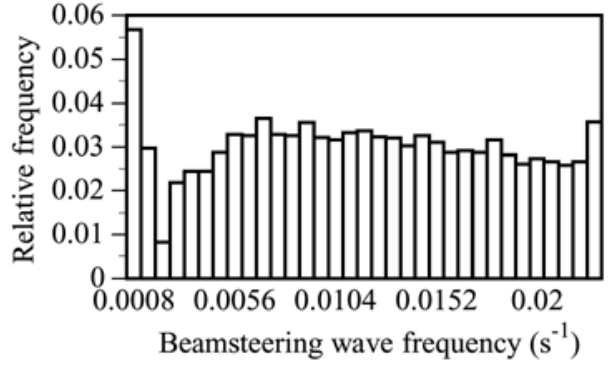

FIG. 11. Frequency of waves detected by the beam-steering.

each block of data. The corresponding cross-spectral phase information was also computed.

\section{a. Beam-steering results from the STABLE II dataset}

A histogram showing the frequency distribution of the waves is presented in Fig. 11. We can see that the number of wave modes detected is roughly uniform for frequencies between 0.005 and $0.023 \mathrm{~s}^{-1}$. The bias toward the greatest number of modes being found in the lowest frequency bin stems from the beam-steering algorithm. Leakage from neighboring bins is used to add weight to the detection of a signal, and so if a signal is chosen as good in a bin, adjacent bins will not be chosen. The peak spectral power is usually detected in the first or second frequency bin. This causes estimates from the third bin, where the minimum occurs in Fig. 11, to be rejected more often than wave estimates occurring in bins of higher frequencies. For similar reasons slightly more wave estimates satisfy the acceptance criteria in the last bin.

Figure 12 shows a histogram of the wave phase speed, $c$, as predicted by the beam-steering routine. Simple arguments suggest that the maximum value of $c$ measurable by the array lies between 25 and $50 \mathrm{~m} \mathrm{~s}^{-1}$. We can see that a relatively small proportion of the detected waves have phase speeds greater than $25 \mathrm{~m} \mathrm{~s}^{-1}$, which is the maximum speed of a wave that the array can sample twice per period. There is a suggestion of two peaks in $c$, centered at $c \sim 6 \mathrm{~m} \mathrm{~s}^{-1}$ and $c \sim 12 \mathrm{~m} \mathrm{~s}^{-1}$, respectively. In the next section, data from these two peaks are investigated for different wave properties.

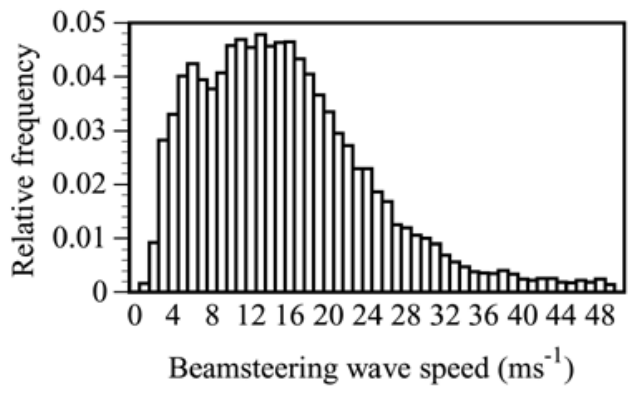

FIG. 12. Phase speed of waves detected by the beam-steering. 


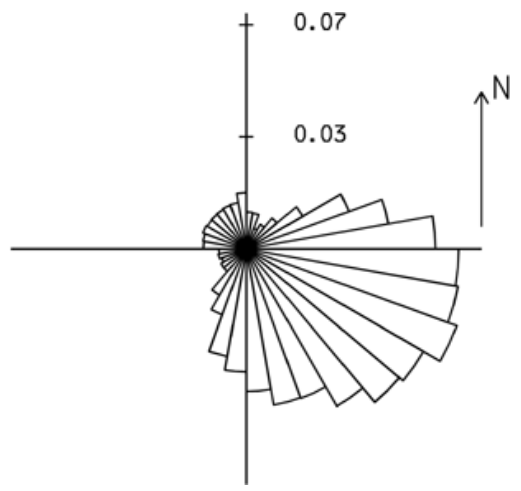

FIG. 13. Propagation direction of waves detected by the beamsteering.

Rees and Mobbs (1988) suggested that the directional consistency of the waves and mean wind direction imply that the majority of waves detected at Halley are topographic in origin. In Fig. 8 we saw that the mean wind was predominantly offshore, from $\sim 80^{\circ}$. In Fig. 13 a polar histogram shows that wave propagation direction is mainly from a southeasterly direction. The distribution of propagation directions is broader than that of mean wind direction, and so there is not a fixed correspondence between wave and wind direction.

The distribution of wavelengths for the waves depicted in Figs. 11 and 12 is shown in Fig. 14. Note the logarithmic spacing of the bins in this figure. We can see that the majority of waves detected by the array have wavelengths of $\sim 800 \mathrm{~m}$. The few results obtained indicating wavelengths in excess of about $5000 \mathrm{~m}$ should be treated with caution due to the limitations imposed by the size of the array.

Figure 15 illustrates the general trend that the coherence of waves averaged over the array is highest for low-frequency waves and decreases with increasing frequency. In Fig. 15 the mean and standard deviation $(\sigma)$ of the coherence are calculated using data from each of 33 frequency bins shown in Fig. 11. Thus the solid line represents the average value from each bin and the dashed lines are $\pm 1 \sigma$ from the mean. This representation is used instead of a two-dimensional histogram for clarity; the distribution of data within each frequency

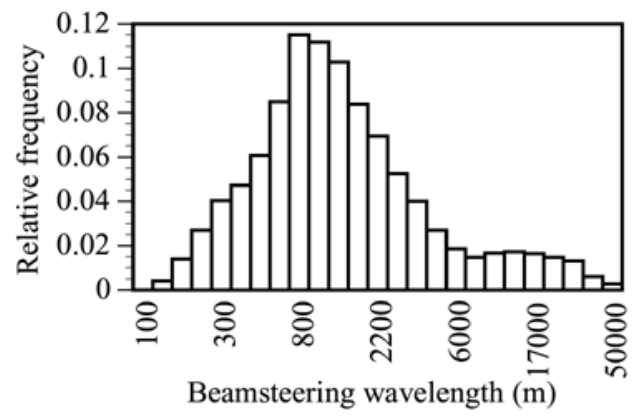

FIG. 14. Wavelengths detected by the beam-steering.

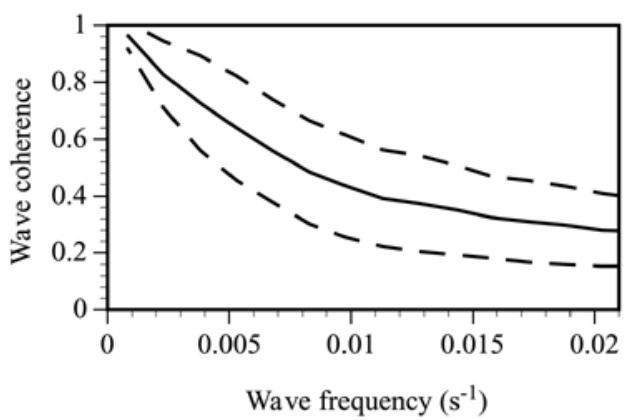

FIG. 15. Coherence of waves detected by the beam-steering vs frequency.

bin has a similar shape, but the mean and "width," represented by $\sigma$, change with frequency. The maximum coherence of $\sim 1$ is found in the lowest frequency bin where it coincides with the minimum $\sigma$. This shows that disturbances with the lowest frequency propagate across the array relatively unchanged compared with higherfrequency modes. Conversely, as higher-frequency modes go through many more cycles while crossing the array, the effects of turbulence are more apparent, resulting in lower coherence. However, the standard deviation $\sigma$ is relatively large at high frequencies in Fig. 15. For waves of frequency $0.02 \mathrm{~s}^{-1}$, the wave coherence may be as high as 0.6 ; thus strongly coherent waves are not limited to the lowest frequencies.

To illustrate the relationship between amplitude and coherence, a histogram of the wave amplitude (on a logarithmic scale) is shown in Fig. 16. A line plot of mean wave coherence associated with each wave amplitude bin is superimposed. The dashed lines indicate one standard deviation $\pm \sigma$ of the coherence from its mean value. There are two peaks in the amplitude distribution. The main peak occurs within the bin corresponding to $\log _{10}$ (wave amplitude $)=-0.2$, where the mean coherence is $\sim 0.5$. The secondary peak is associated with much larger amplitude waves $\log _{10}$ (wave amplitude $)=2.5$. For these waves the coherence is close to the maximum value of 1.0, indicating that largeramplitude waves tend to propagate across the array without change of form. The smaller-amplitude waves, par-

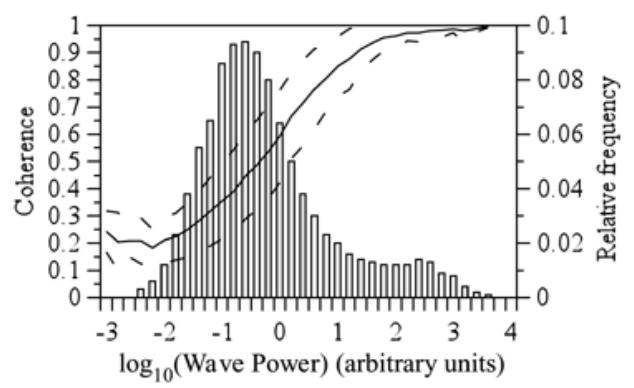

FIG. 16. Histogram of wave amplitude and a plot of coherence vs wave amplitude. 


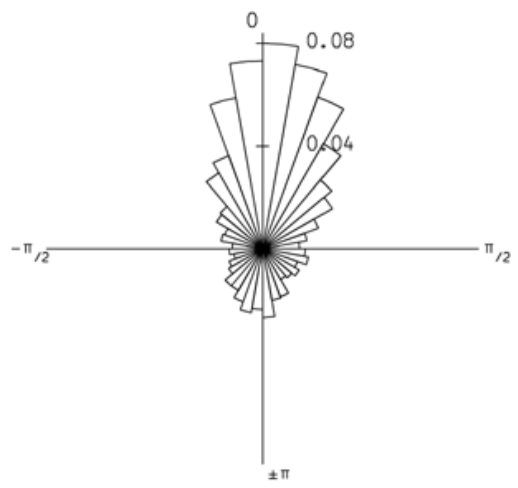

FIG. 17. $\mathrm{Ph}(u p)$ using 4-m sonic $u$ with $\operatorname{Co}(u p) \geq 0.05$.

ticularly those for which nonlinear effects can be ignored, are more likely to be affected by the mean flow and turbulence. Thus the smaller-amplitude waves are associated with a lower coherence.

\section{b. Cross-spectral phase}

Cross-spectral phases and coherence were calculated for each beam-steering wave estimate. Following Rees (1991), results are restricted to data with coherence greater than 0.05 . In this section wind speed data are taken from the sonic anemometer at $4 \mathrm{~m}$ on the mast in Fig. 2. This is the closest sonic to the buried microbarograph and therefore least affected by vertically tilting phase lines.

The information presented in Table 1 indicates that the cross-spectral phase between horizontal wind speed and pressure fluctuations $\mathrm{Ph}(u p)=0, \pi$ for IGWs, $= \pm \pi$ for Kelvin-Helmholtz instabilities, and takes an indeterminate value when turbulent motions dominate the flow. In Fig. 17, $\mathrm{Ph}(u p)$ is plotted for the beam-steering results with values of the cross-spectral coherence $\mathrm{Co}($ up) greater than 0.05 . Of the 13688 estimates obtained by the beam-steering routine, 6687 satisfied this criterion. We can see from the figure that the crossspectral phases are concentrated around $\mathrm{Ph}(u p)=0$, with a secondary maximum around $\mathrm{Ph}(u p)=\pi$. A relatively small number of detected waves had an intermediate phase. The maxima are broadened by the action of turbulence on the waves and by phase shifts due to the $\sim 4 \mathrm{~m}$ vertical separation of the two instruments. These results suggest strongly that the waves detected by the beam-steering analysis are IGWs, rather than pressure fluctuations due to density inhomogeneities drifting across the array with the mean wind (as postulated by Hauf et al. 1996). In the latter case the phase would not be expected to be split into two categories. Since any measured fluctuations in $u$ would be turbulent in nature, they would decorrelate rapidly across the array. Fluctuations in $p$ would be more regular, determined by the density inhomogeneities. Therefore the two signals would necessarily have a random phase difference, determined by the local turbulence.

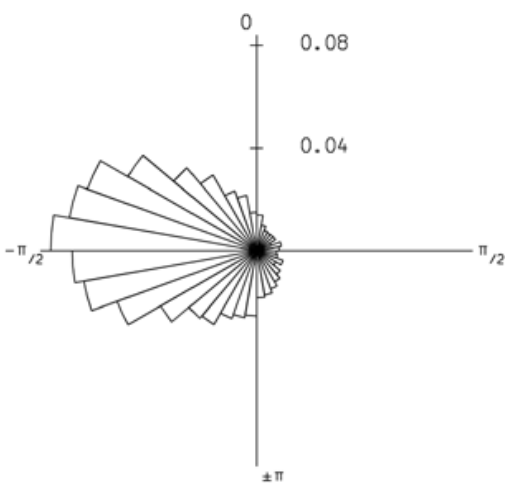

FIG. 18. $\mathrm{Ph}(w p)$ using $4-\mathrm{m}$ sonic $w$ with $\operatorname{Co}(w p) \geq 0.05$.

In Fig. 18 a polar histogram of $\mathrm{Ph}(w p)$ is plotted for all waves with a value of the cross coherence $\operatorname{Co}(w p)$ $\geq 0.05$. In the presence of IGWs, $\mathrm{Ph}(w p)= \pm \pi / 2$. We can see that the distribution of $w p$ phase for the waves is predominantly centered around $-\pi / 2$, which is indicative of IGWs traveling faster than the mean wind at $4 \mathrm{~m}$. This result is consistent with the findings of Rees and Mobbs (1988), who used a linear model to show that most of the wave activity at Halley can be attributed to the presence of trapped, neutral modes, generated at or near the surface. It is reasonable to expect that the propagation of such modes would be controlled by the near-surface wind.

We would expect the $w T$ cross-spectral phases to be 0 or $\pi$ when turbulent fluctuations dominate the wind measurements. This was found to be the case: $\mathrm{Ph}(w T)$ is plotted in Fig. 19 for records when the stability as measured by the bulk Brunt-Väisälä frequency at $10 \mathrm{~m}$ $N_{\text {bv }} \leq 0.06 \mathrm{~s}^{-1}$. Note that $N_{\text {bv }}$ is defined in an analogous manner to the bulk Richardson number, $\mathrm{Ri}_{b}$. The $N_{\text {bv }}$ at $10 \mathrm{~m}$ is calculated using the temperature difference between 10 and $4 \mathrm{~m}$. The fact that we have found a "cutoff" stability level, which essentially separates when IGWs or turbulent eddies dominate the flow, enhances the findings of Rees (1991), who found that turbulence phases were observed when the gradient Richardson number fell below 0.25 .

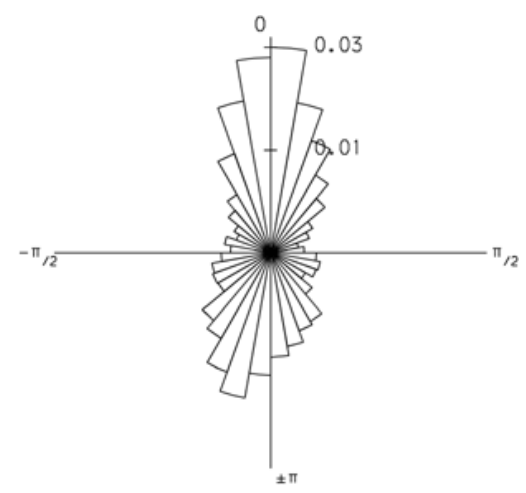

FIG. 19. $\mathrm{Ph}(w T)$ using $4-\mathrm{m}$ sonic $w$ for low stability and $\operatorname{Co}(w T \geq$ $0.05)$ 


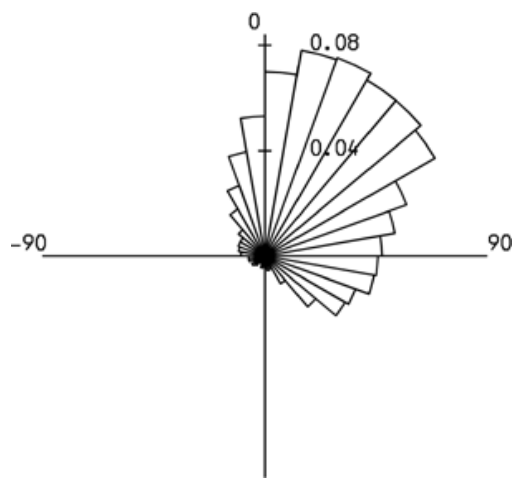

FIG. 20. Difference between wave and wind direction at $32 \mathrm{~m}$ (in degrees).

\section{c. Differences between mean wind and wave speed and direction}

Differences between the direction and speed of the mean wind and the propagation direction and phase speed of the detected waves can be used to further examine the properties of the waves. If we are able to classify, in some way, the differences between the wind velocity and the speed and direction of the waves, we may be able to gain insight into the contribution the waves make to the boundary layer fluxes. Rees and Mobbs (1988) showed that a significant proportion of the waves at Halley are trapped, neutral modes generated at or near the surface. Thus it is likely that their propagation is controlled by the near-surface wind. We have therefore attempted to categorize the waves initially according to their propagation direction relative to the mean wind. Following on from this, the crossspectral phases belonging to subdivisions categorized by wave speed have been analyzed. In Fig. 20 the difference between the direction of wave propagation and mean wind at $32 \mathrm{~m}, \delta \theta$, is plotted. In this figure the angular scale is in degrees, in steps of $10^{\circ}$, with zero difference in the "northerly" direction. Positive deviations are indicated in a clockwise sense (waves veered from the mean wind) and negative deviations counterclockwise (waves backed from the mean wind). The number of data points available for this figure was 10875 ; this is less than the maximum 13688 beamsteering estimates because the 32-m sonic anemometer was occasionally inoperative. It is evident that the majority of waves detected by the beam-steering are veered from the mean wind by $0^{\circ}-60^{\circ}$. There are a significant number with extreme veering $\left(>90^{\circ}\right.$, where the mean wind opposes the wave) and somewhat fewer backed waves. These categories (backed, veered, and opposing) lead to a convenient categorization of the waves, which is explored in the next section.

Figure 21 shows the difference between the wave phase speed, $c$, and the mean wind speed at $32 \mathrm{~m}, \bar{u}$. The difference between $c$ and the mean wind speed resolved parallel to the wave propagation direction, $c-$

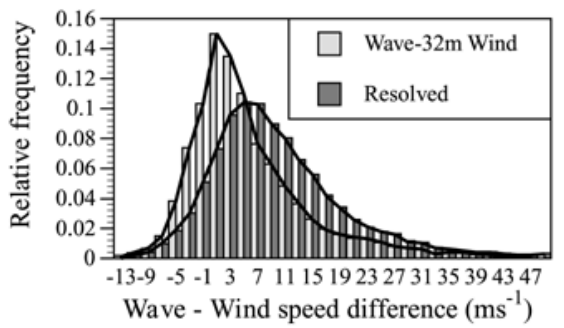

FIG. 21. Difference between wave speed and wind speed at $32 \mathrm{~m}$.

$\bar{u} \cos (\delta \theta)$, is also plotted. The midpoints of the bins are separated by $2 \mathrm{~m} \mathrm{~s}^{-1}$. The shapes of the two distributions are emphasised by lines joining the midpoints of each category. It is evident from the histogram of $c-\bar{u}$ that waves detected by the beam-steering tend to propagate faster than the mean wind. Wave speeds exceed wind speeds by an average of $6.6 \mathrm{~m} \mathrm{~s}^{-1}$. However, the distribution is skewed such that mode of the difference in speeds is just $1 \mathrm{~m} \mathrm{~s}^{-1}$, indicating that a significant proportion of the waves do propagate at speeds close to the $32 \mathrm{~m}$ wind speed. A small number of waves travel more slowly than the $32-\mathrm{m}$ wind. Although it is common practice to compare wave speeds with the mean wind speed (e.g., Rees and Mobbs 1988), it is perhaps more appropriate to examine the wave speed in relation to the component of the wind in the direction of wave propagation. Unless the wave direction and wind direction are the same for all sections of data, we would expect the peak on this histogram to indicate that $c-\bar{u} \cos (\delta \theta)$ is typically greater than $c-\bar{u}$ [remember that in the vast majority of cases the wave propagation direction is veered by up to $90^{\circ}$ from the mean wind direction (Fig. 20), so that $\cos (\delta \theta) \leq 1$ ]. A narrow distribution focused around zero value of $c-\bar{u} \cos (\delta \theta)$ would indicate that the waves are strongly influenced by the local environment. As expected, in the histogram of $c-\bar{u}$ $\cos (\delta \theta)$, the peak has been displaced toward the right. The mean difference between the phase speed and the wind speed is now $11.0 \mathrm{~m} \mathrm{~s}^{-1}$, with the mode of the difference being $5 \mathrm{~m} \mathrm{~s}^{-1}$. The distribution is also broader; the standard deviation of the data used in the histogram increases from 8.8 to $9.9 \mathrm{~m} \mathrm{~s}^{-1}$ for the resolved data. From these results we can deduce that although about $10 \%$ of the wave activity at Halley is strongly controlled by the 32-m wind (i.e., by conditions near the top of the boundary layer), by far the majority of waves remain relatively uninfluenced by the local conditions at this level. Again this result is in agreement with the earlier findings of Rees and Mobbs (1988), who claimed that a significant proportion of the waves at Halley are generated at or near the ground.

\section{Waves categorized by propagation direction relative to the mean wind}

To investigate further the properties of the detected waves, results are presented in this section from data 

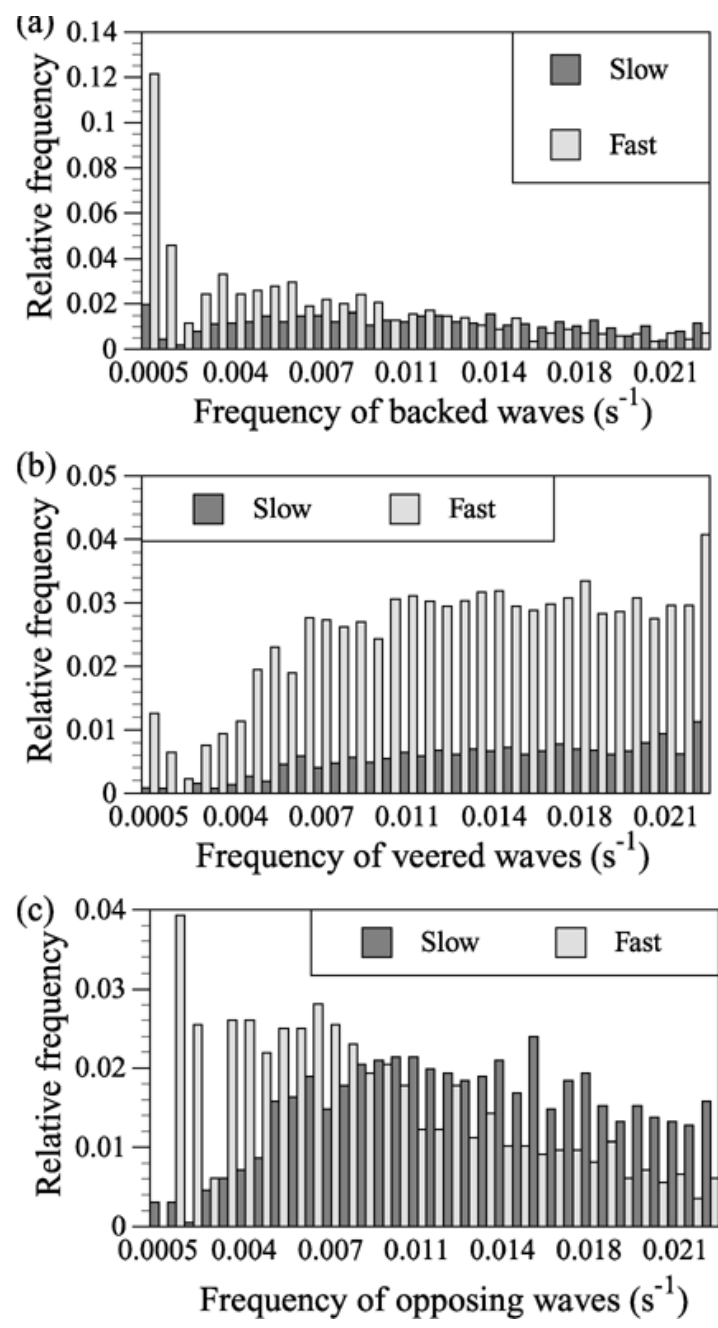

FIG. 22. Wave frequency for (a) backed, (b) veered, and (c) opposing waves. In each case results have been subdivided into two further categories corresponding to slow waves and fast waves.

separated into categories based upon the differences between the wave direction and the wind direction at 32 $\mathrm{m}, \delta \theta$, as shown in Fig. 20. The categories are "backed" $\left(-90^{\circ} \leq \delta \theta<0^{\circ}\right)$, "veered" $\left(0^{\circ} \leq \delta \theta<90^{\circ}\right)$, and "opposing" $\left(270^{\circ}>\delta \theta \geq 90^{\circ}\right)$. We further subdivide the data according to the wave phase speed. In Fig. 12 there is the suggestion of an approximately bimodal distribution, with peaks at $c \sim 6 \mathrm{~m} \mathrm{~s}^{-1}$ and $c \sim 12 \mathrm{~m}$ $\mathrm{s}^{-1}$. Thus we separate the data into the following subdivisions: (i) "slow" waves with $c=0-9 \mathrm{~m} \mathrm{~s}^{-1}$ and (ii) "fast" waves with $c=9-25 \mathrm{~m} \mathrm{~s}^{-1}$.

In Fig. 22 histograms of wave frequency are plotted for the three categories of $\delta \theta$, subdivided into fast and slow waves. The data in each part of the figure are plotted relative to the total number of points in the backed, veered, and opposing categories, respectively. The total number of points in each category and the percentage of fast and slow waves is shown in Table 2. Note that the sum of the percentages in each column is
TABLE 2. Table showing the total number of detected waves shown in Fig. 20.

\begin{tabular}{lccc}
\hline \hline & $\begin{array}{c}\text { Backed } \\
-90^{\circ} \leq \delta \theta<0^{\circ}\end{array}$ & $\begin{array}{c}\text { Veered } \\
0^{\circ} \leq \delta \theta<90^{\circ}\end{array}$ & $\begin{array}{c}\text { Opposing } \\
270^{\circ}>\delta \theta \geq 90^{\circ}\end{array}$ \\
\hline Slow & $31 \%$ & $15 \%$ & $45 \%$ \\
Fast & $52 \%$ & $69 \%$ & $46 \%$ \\
Total & 2459 & 6274 & 2142 \\
\hline
\end{tabular}

less than $100 \%$ as wave speeds in excess of $25 \mathrm{~m} \mathrm{~s}^{-1}$ have been neglected due to possible uncertainty in the results arising from the limitations of the array. For all three categories the peaks in the histogram for fast waves are associated with the lowest frequencies (i.e., less than $0.004 \mathrm{~s}^{-1}$ ). This indicates that there is no systematic relationship between the direction of these lowfrequency, fast waves and the 32-m wind. It is therefore likely that such waves are generated either aloft [possibly in layers of dynamical instability; King et al. (1987); Rees (1987)] or in the boundary layer some distance from Halley. This result is consistent with the work of Einaudi et al. (1989), who found that local conditions in the boundary layer had little influence on longer-period waves. More than half of the waves detected fell into the category of veered waves. From Fig. $22 \mathrm{~b}$ it can be seen that the majority of these waves are fast waves with frequencies greater than $0.004 \mathrm{~s}^{-1}$. These are likely to be mainly the horizontally propagating, neutral modes identified by Rees and Mobbs (1988). Figure 22c indicates that for the case of waves propagating in a direction opposing that of the mean wind, those of lower frequency tend to be mainly fast waves, but slow waves dominate at higher frequencies.

As a measure of the underlying atmospheric stability, histograms of bulk $N_{\text {bv }}$ for each of the categories in Fig. 22 are shown in Fig. 23. Notice that for each category the distributions are similar. Faster waves tend to be associated with $N_{\text {bv }}<0.07 \mathrm{~s}^{-1}$. Peaks in the histograms for the slow waves occur for $N_{\mathrm{bv}} \sim 0.09-0.11 \mathrm{~s}^{-1}$, that is, when the stratification is more stable. These results are consistent with those shown earlier in Figs. 9 and 10 that summarized the mean atmospheric conditions at Halley IV station. Stronger offshore winds were associated with weaker stratifications than onshore winds. As we have demonstrated that horizontally propagating trapped modes with phase speeds greater than the mean wind speed are commonplace when offshore winds occur, we would expect that generally faster wave speeds would be associated with less strong stratification.

The cross-spectral phase of the backed waves also differs from those in the veered and opposing categories. In particular, we saw from Fig. 17 that the majority of waves detected corresponded with $\mathrm{Ph}(u p) \approx 0$, with a small number of waves with $\mathrm{Ph}(u p) \approx \pi$. In Fig. 24, $\mathrm{Ph}(u p)$ (using the 4-m sonic $u$ ) is plotted for all backed waves with $\operatorname{coh}^{2}(u p) \geq 0.05$. Each bin is plotted as a fraction of the total data plotted in Fig. 17. From this we can see that over $30 \%$ of waves with $\operatorname{Ph}(u p) \approx \pi$ 

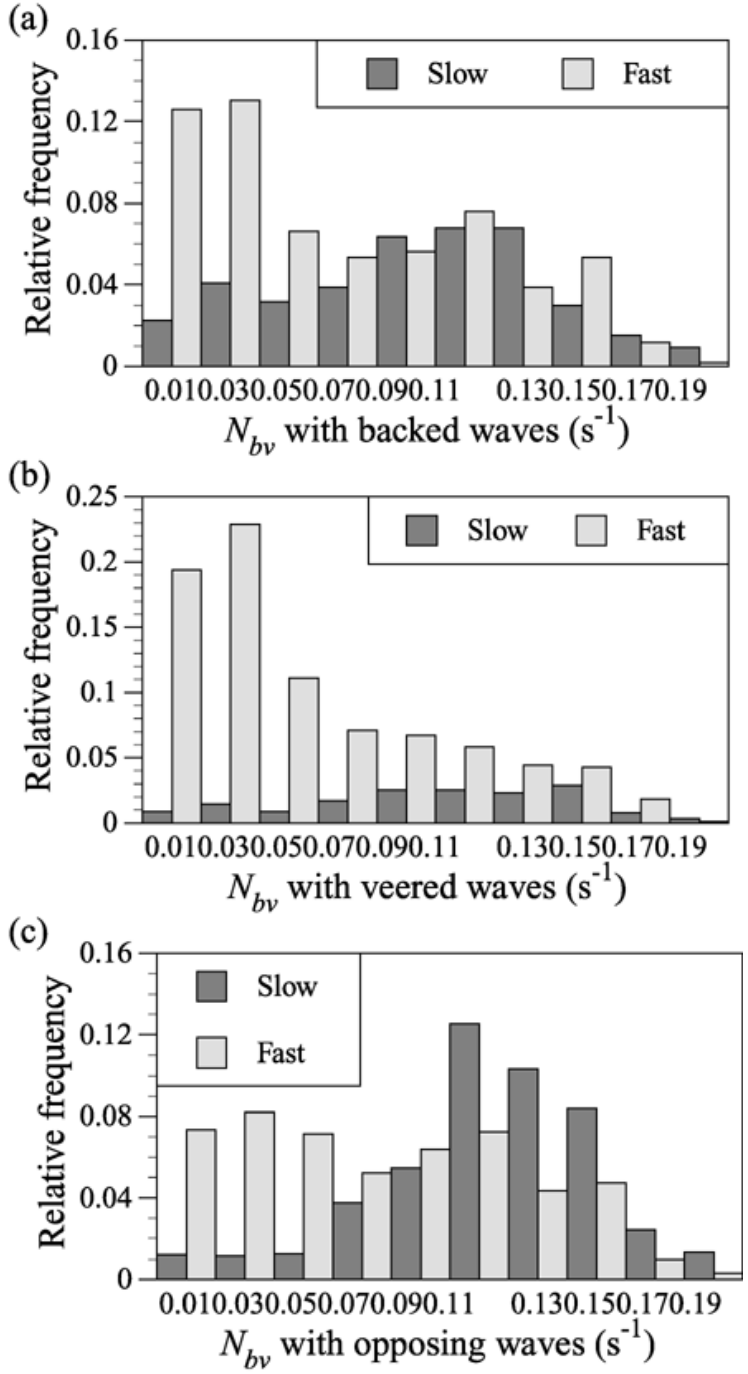

FIG. 23. 10-m bulk atmospheric stability for situations when (a) backed, (b) veered, and (c) opposing waves are observed. Results have been subdivided into slow waves and fast waves.

correspond with backed waves, even though they compose only $17 \%$ of the total waves detected. Around $55 \%$ of all backed waves have $\operatorname{Ph}(u p) \approx \pi$; the remainder have $\operatorname{Ph}(u p) \approx 0$. In contrast, within the veered and opposing wave categories only $24 \%$ have $\operatorname{Ph}(u p) \approx \pi$.

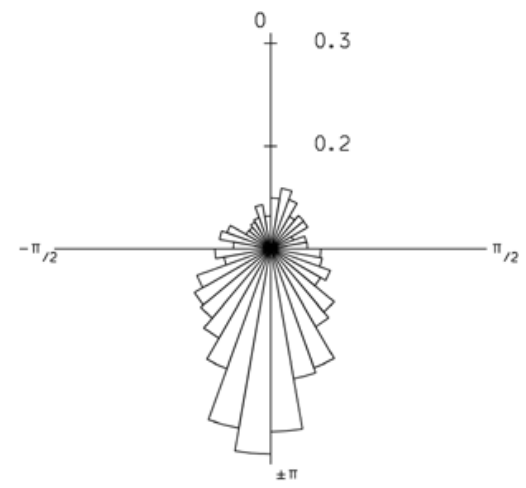

FIG. 24. $\mathrm{Ph}($ up $)$ using 4-m sonic speed for backed waves with $\operatorname{Co}(u p) \geq 0.05$.

In both cases these proportions are similar for fast and slow waves.

\section{Distributions of wave minus wind speed}

Histograms of the wave phase speed minus the 32-m mean wind speed for all the wave data were plotted in Fig. 21. When the data are subdivided into the categories under discussion, the shapes of the distributions remain similar. They can therefore be categorized by a mean value and some measure of their width, which is taken here to be the standard deviation, $\sigma$, of the underlying data. Data for the slow and fast waves from the three directional categories are shown in Table 3.

These data illustrate further how the properties of waves in each category depend differently on the mean wind at $32 \mathrm{~m}$. In the case of waves backed from the mean wind, $\sigma$ is comparable to the value obtained from all of the data (i.e., that shown in Fig. 21), which is large compared to the other two categories and comparable to the mean value of $c-\bar{u} \cos \delta \theta$. This indicates that backed waves are influenced the least by local winds in the boundary layer. In contrast, the phase speed of opposing waves is, on average, much faster than the mean wind (not unexpected for propagation in opposing conditions), and the standard deviation, $\sigma$, is relatively small compared to the mean value of $c-\bar{u} \cos \delta \theta$. This indicates that opposing wave speeds are influenced by the mean wind speed in the boundary layer. The veered waves show behavior intermediate between the other

TABLE 3. Table showing the mean and standard deviation $(\sigma)$ of the difference between the wave phase speed and the mean wind speed $\left(\mathrm{m} \mathrm{s}^{-1}\right)$ at $32 \mathrm{~m}$ resolved parallel to the wave direction.

\begin{tabular}{lcccc}
\hline \hline Wave category & Total & $\begin{array}{c}\text { Backed } \\
-90^{\circ} \leq \delta \theta<0^{\circ}\end{array}$ & $\begin{array}{c}\text { Veered } \\
0^{\circ} \leq \delta \theta<90^{\circ}\end{array}$ & $270^{\circ}>\delta \theta \geq 90^{\circ}$ \\
\hline Slow & & & & \\
$\quad$ Mean & 4.5 & 1.3 & 2.8 & 8.6 \\
$\sigma$ & 4.5 & 4.2 & 2.6 & 2.6 \\
Fast & & & 8.6 & 17.8 \\
Mean & 10.6 & 8.0 & 5.8 & 5.1 \\
$\sigma$ & 7.6 & 8.8 & & \\
\hline
\end{tabular}


cases. The slow, veered waves have relatively large $\sigma$ compared to the mean, indicating a small dependence on $\bar{u}$. For the fast, veered waves, however, $c-\bar{u} \cos \delta \theta$ has a relatively smaller spread indicating that the resolved wind component exerts a greater influence on these waves than on the slow, veered waves.

These results support the conclusions that (i) opposing waves are substantially controlled by the waveguide and (ii) backed and veered waves are generally much less affected by conditions within the boundary layer at Halley.

\section{Conclusions}

An extensive dataset was compiled from observations of wind, temperature, and surface pressure fluctuations from Halley IV station, Antarctica, as part the the British Antarctic Survey's STABLE project aimed at investigating the properties of the stably stratified atmospheric boundary layer. Internal gravity waves are characteristic of the stable boundary layer. A climatology for the gravity wave activity in the boundary layer overlying Halley IV station has been constructed using obervations from an instrumented meteorological mast and a specially developed microbarograph array. Data analysis techniques included a beam-steering routine to determine wave parameters from the microbarograph data and cross-spectral analysis to identify the nature of fluctuations in the field variables.

The wind regime at Halley is essentially bimodal. The predominant wind direction is easterly. Strong easterly winds occur frequently due to synoptic and katabatic forcings acting together. The secondary peak corresponds to relatively slower winds blowing toward Halley from the sea. The boundary layer at Halley was usually found to be more strongly stably stratified over the lowest $32 \mathrm{~m}$ when onshore winds prevailed.

The microbarographs detected the presence of coherent motions in the period range 1-20 min throughout the 9-month observational campaign. A spectral gap (at a period of around $34 \mathrm{~min}$ ) was found to exist between the synoptic variations responsible for the mean trend and the internal gravity waves of interest. Analysis of cross-spectral phases indicated that the coherent fluctuations could be attributed to propagating internal gravity waves [rather than drifting density inhomogeneities as in the study of Hauf et al. (1996)]. Furthermore, strong coherence between the sensors was found to occur nearly all of the time. Such activity was also reported by Einaudi et al. (1989) and is again in contrast with the study of Hauf et al. (1996), who found that coherent motions persisted for at most five or six cycles.

Root-mean-square amplitudes of $24 \mu \mathrm{b}$ were typical for the waves at Halley, but a number of isolated events with amplitudes of up to $180 \mu \mathrm{b}$ were also detected. Most of the waves were found to propagate at speeds in excess of the local mean wind speed, and over half of the wave events were veered by about $60^{\circ}$ from the mean wind direction. In order to investigate further the properties of the waves, observations of approximately 3-h duration were categorized according to wave speed ("slow" waves were defined as propagating with phase speeds of 0-9 $\mathrm{m} \mathrm{s}^{-1}$ and "fast" waves 9-25 $\mathrm{m} \mathrm{s}^{-1}$ ) and direction relative to the mean wind direction at $32 \mathrm{~m}$ (veered, backed, and opposing). It was deduced that about $10 \%$ of all wave activity was strongly controlled by conditions near the top of the boundary layer. The lowest-frequency waves (i.e., less than $0.004 \mathrm{~s}^{-1}$ ) were predominantly fast waves. No systematic relationship was found between the properties of these waves and the local wind. It is therefore likely that such waves are generated in regions aloft, possibly in layers of dynamical instability. This finding is in agreement with Einaudi et al. (1989), who deduced that local conditions in the boundary layer exerted little influence on longer-period waves. Most of the higher-frequency (i.e., greater than $0.004 \mathrm{~s}^{-1}$ ) fast waves at Halley were veered to the mean wind. These waves correspond to the trapped neutral modes described by Rees and Mobbs (1988). These fast waves were also associated with strong offshore winds and comparatively weaker temperature inversions (compared to those measured when onshore winds prevailed). Thus it is reasonable to deduce that these waves were generated in the katabatic flow regime near the Hinge Line, where the ice shelf meets the Antarctic continent.

The climatology presented in this paper will provide the basis for future studies using the STABLE dataset. A study of the largest-amplitude wave events, often associated with the passing of warm air fronts, is currently being undertaken.

Acknowledgments. We would like to thank Dr. S. D. Mobbs for his involvement in the planning of the microbarograph experiment. JMR and JCWP would like to acknowledge the NERC for the provision of Research Grant GR3/09321. JMR is grateful to the North Atlantic Treaty Organisation for Grant CRG940242.

\section{REFERENCES}

Anderson, P. S., S. D. Mobbs, J. C. King, I. McConnell, and J. M. Rees, 1992: A microbarograph for internal gravity wave studies in Antarctica. J. Antarct. Sci., 4, 241-248.

Bull, G., R. Dubois, J. Neisser, and J. G. Stangenberg, 1981: Untersuchungen uber Schwerewellen in Gebirgshe. Z. Meteor., 31, 267-279.

Capon, J., 1969: High-resolution frequency-wavenumber spectrum analysis. Proc. IEEE, 57, 1408-1416.

Cheung, T. K., and C. G. Little, 1990: Meteorological tower, microbarograph array, and sodar observations of solitary-like waves in the nocturnal boundary layer. J. Atmos. Sci., 47, 2516-2536.

Denholm-Price, J. C. W., and J. M. Rees, 1998: A practical example of low-frequency trend removal. Bound.-Layer Meteor., 86, 181187.

- , and — 1999: Detecting waves using an array of sensors. Mon. Wea. Rev., 127, 57-69.

Egger, J., C. Wamser, and C. Kottmeier, 1993: Internal atmospheric gravity waves near the coast of Antarctica. Bound.-Layer Meteor., 66, 1-17. 
Einaudi, F., A. J. Bedard, and J. J. Finnigan, 1989: A climatology of gravity waves and other coherent disturbances at the Boulder Atmospheric Observatory during March-April 1984. J. Atmos. Sci., 46, 303-329.

Gedzelman, S. D., 1983: Short-period atmospheric gravity waves: A study of their statistical properties and source mechanisms. Mon. Wea. Rev., 111, 1293-1299.

Haubrich, R. A., 1968: Array design. Bull. Seismol. Soc. Amer., 58, 977-991.

Hauf, T., U. Finke, J. Neisser, G. Bull, and J.-G. Stangenberg, 1996 : A ground-based network for atmospheric pressure fluctuations. J. Atmos. Oceanic Technol., 13, 1001-1023.

Hooke, W. H., J. M. Young, and D. W. Beran, 1972: Atmospheric waves observed in the planetary boundary layer using an acoustic sounder and a microbarograph array. Bound.-Layer Meteor., 2, 371-380.

Keliher, T. E., 1975: The occurrence of microbarograph-detected gravity waves compared with the existence of dynamically unstable wind shear layers. J. Geophys. Res., 80, 2967-2976.

Kikuchi, T., 1988: A case study of a wave-like cloud and gravity wave in the lower troposphere in Mizuho Plateau Antarctica. Bound.-Layer Meteor., 43, 403-409.

King, J. C., 1989: Low-level wind profiles at an Antarctic coastal station. J. Antarct. Sci., 1, 169-178.

- 1990: Some measurements of turbulence over an Antarctic ice shelf. Quart. J. Roy. Meteor. Soc., 116, 379-400.
— 1993: Control of near-surface winds over an Antarctic ice shelf. J. Geophys. Res., 98, 12 949-12 953.

— - S. D. Mobbs, M. S. Darby, and J. M. Rees, 1987: Observations of an internal gravity wave in the lower troposphere at Halley, Antarctica. Bound.-Layer Meteor., 39, 1-13.

- - - J. M. Rees, P. S. Anderson, and A. D. Culf, 1989: The Stable Antarctic Boundary Layer Experiment at Halley Base. Weather, 44, 398-405.

Merrill, J. T., 1977: Observational and theoretical study of shear instability in the airflow near the ground. J. Atmos. Sci., 34, 911921.

Rees, J. M., 1987: The propagation of internal gravity waves in the stably stratified atmospheric boundary layer. Ann. Geophys., 5B, 421-432.

- 1991: On the characteristics of eddies in the stable atmospheric boundary layer. Bound.-Layer Meteor., 55, 325-343.

_ , and S. D. Mobbs, 1988: Studies of internal gravity waves at Halley Station, Antarctica, using wind observations. Quart. J. Roy. Meteor. Soc., 114, 939-966.

Smart, E., and E. A. Flinn, 1971: Fast frequency-wavenumber analysis and Fisher signal detection in real-time infrasonic array data processing. Geophys. J. Roy. Astrophys. Sci., 26, 279-284.

Yägue, C., and J. L. Cano, 1994: The influence of stratification on heat and momentum transfer in Antarctica. Bound.-Layer Meteor., 69, 123-136. 